\title{
Epidemiological and clinical insights from SARS-CoV-2 RT-PCR crossing threshold values, France, January to November 2020
}

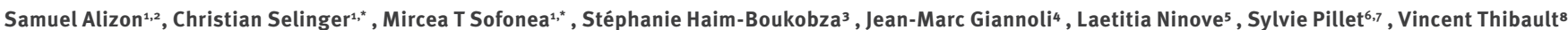
, Alexis de Rougemont ${ }^{9,10}$, Camille Tumiotto ${ }^{11}$, Morgane Solis ${ }^{12}$, Robin Stephan ${ }^{13}$, Céline Bressollette-Bodin ${ }^{14}$, Maud Salmona ${ }^{15}$, Anne-Sophie L'Honneur ${ }^{16}$, Sylvie Behillil $^{17}$, Caroline Lefeuvre ${ }^{18,19}$, Julia Dina ${ }^{20}$, Sébastien Hantz ${ }^{21,22}$, Cédric Hartard ${ }^{23}$, David Veyer ${ }^{24}$, Héloïse M Delagrèverie ${ }^{25}$, Slim Fourati ${ }^{26}$, Benoît Visseaux ${ }^{27}$,

Cécile Henquell $^{28}$, Bruno Lina ${ }^{29}$, Vincent Foulongne ${ }^{30}$, Sonia Burrel ${ }^{31}$, on behalf of the SFM COVID-19 study group ${ }^{32}$

1. MIVEGEC, CNRS, IRD, Université de Montpellier, France

2. Center for Interdisciplinary Research in Biology (CIRB), College de France, CNRS, INSERM, Université PSL, Paris, France

3. Laboratoire CERBA, Saint-Ouen-L'Aumône, France

4. BIOGROUP, Scientific Direction, Lyon, France

5. Unité des Virus Émergents (UVE: Aix-Marseille Univ-IRD 190-Inserm 1207-IHU Méditerranée Infection), Marseille, France

6. Laboratoire des agents infectieux et d'hygiène, CHU de Saint-Etienne, France

7. CIRI, Centre International de Recherche en Infectiologie, GIMAP team, University of Lyon, University of Saint-Etienne, INSERM, U1111, CNRS UMR5308, ENS de Lyon, UCBL, Lyon, France

8. Laboratoire de Virologie, CHU Rennes, Rennes, France

9. Laboratory of Virology-Serology, University Hospital of Dijon Bourgogne, Dijon, France

10. UMR PAM A 02.102 Procédés Alimentaires et Microbiologiques, Université de Bourgogne Franche-Comté/AgroSup Dijon, Dijon, France

11. University of Bordeaux, CNRS-UMR 5234, CHU Bordeaux, Virology Department, Bordeaux, France

12. CHU de Strasbourg, Laboratoire de Virologie, Strasbourg, France, Université de Strasbourg, INSERM, IRM UMR_S 1109, Strasbourg, France

13. Laboratoire de Microbiologie, CHU Nîmes, Nîmes, France

14. CHU Nantes, Nantes Université, Service de Virologie, Nantes, France

15. 15 Laboratoire de Virologie, Hôpital Saint Louis, APHP, INSERM U976, équipe INSIGHT, Université de Paris, Paris, France

16. Assistance Publique-Hôpitaux de Paris, Hôpital Cochin, Service de Virologie, Paris, France

17. 17 National Reference Center for Respiratory Viruses, Molecular Genetics of RNA Viruses, UMR 3569 CNRS, University of Paris, Institut Pasteur, Paris, France

18. Département de Biologie des Agents Infectieux, Laboratoire de Virologie, CHU d’Angers, Angers, France

19. Laboratoire HIFIH, UPRES EA 3859, Université d'Angers, Angers, France

20. Laboratoire de Virologie, CHU de Caen, UNICAEN, INSERM U1311 DYNAMICURE, Université de Caen Normandie, Caen, France

21. CHU Limoges, Laboratoire de Bactériologie-Virologie-Hygiène, Limoges, France

22. RESINFIT, U 1092, University of Limoges, Limoges, France

23. Laboratoire de Virologie, CHRU de Nancy Brabois, Vandoeuvre-lès-Nancy, France; Université de Lorraine, CNRS, LCPME, Nancy, France

24. Laboratoire de Virologie, Service de Microbiologie, hôpital européen Georges Pompidou, Assistance Publique-Hôpitaux de Paris et Unité de Génomique Fonctionnelle des Tumeurs Solides, Centre de Recherche des Cordeliers, INSERM, Université Paris, Paris, France.

25. AP-HP, Hôpital Avicenne, Laboratoire de microbiologie clinique, Bobigny, France

26. Henri Mondor Hospital, virology department, Créteil, France

27. Université de Paris, Inserm, UMR 1137 IAME et Laboratoire de Virologie, Hôpital Bichat Claude Bernard, AP-HP, Paris, France

28. Service de Virologie médicale, 3IHP, CHU Clermont-Ferrand, Clermont-Ferrand, France

29. CNR des virus des infections respiratoires (dont la Grippe), Institut des Agents Infectieux, Hopital de la Croix Rousse, HCL, Lyon, France

30. Pathogenesis and control of chronic and emerging infections, Université de Montpellier, UMR 1058, CHU de Montpellier, Inserm, Université des Antilles, Montpellier, France

31. Sorbonne Université, INSERM U1136, Institut Pierre Louis d’Epidémiologie et de Santé Publique (IPLESP), AP-HP, Hôpital Pitié-Salpêtrière, Service de Virologie, Paris, France

32. French Society of Microbiology (SFM), https://www.sfm-microbiologie.org

* These authors contributed equally to this work.

Correspondence: Samuel Alizon (samuel.alizon@cnrs.fr)

Citation style for this article:

Alizon Samuel, Selinger Christian, Sofonea Mircea T, Haim-Boukobza Stéphanie, Giannoli Jean-Marc, Ninove Laetitia, Pillet Sylvie, Thibault Vincent, de Rougemont Alexis, Tumiotto Camille, Solis Morgane, Stephan Robin, Bressollette-Bodin Céline, Salmona Maud, L'Honneur Anne-Sophie, Behillil Sylvie, Lefeuvre Caroline, Dina Julia, Hantz Sébastien, Hartard Cédric, Veyer David, Delagrèverie Héloïse M. Fourati Slim, Visseaux Benoît, Henquell Cécile, Lina Bruno, Foulongne Vincent, Burrel Sonia, on behalf of the SFM COVID-19 study group. Epidemiological and clinical insights from SARS-CoV-2 RT-PCR crossing threshold values, France, January to November 2020 . Euro Surveill. 2022;27(6):pii=2100406. https://doi.org/10.2807/1560-7917.ES.2022.27.6.2100406

Article submitted on 31 Mar 2021 / accepted on 15 Oct 2021 / published on 10 Feb 2022

Background: The COVID-19 pandemic has led to an unprecedented daily use of RT-PCR tests. These tests are interpreted qualitatively for diagnosis, and the relevance of the test result intensity, i.e. the number of quantification cycles (Cq), is debated because of strong potential biases. Aim: We explored the possibility to use $\mathrm{Cq}$ values from SARS-CoV-2 screening tests to better understand the spread of an epidemic and to better understand the biology of the infection. Methods: We used linear regression models to analyse a large database of $793,479 \mathrm{Cq}$ values from tests performed on more than 2 million samples between 21 January and 30 November 2020, i.e. the first two pandemic waves. We performed time series analysis using autoregressive integrated moving average (ARIMA) models to estimate whether Cq data information improves short-term predictions of epidemiological dynamics. Results: Although we found that the $\mathrm{Cq}$ values varied depending on the testing laboratory or the assay used, we detected strong significant trends associated with patient age, number of days after symptoms onset or the state of the epidemic (the temporal reproduction number) at the time of the test. Furthermore, knowing the quartiles of the Cq distribution greatly reduced the error in predicting the temporal reproduction number of the COVID-19 epidemic. Conclusion: Our results suggest that $\mathrm{Cq}$ values of screening tests performed in the general population 
Main factors affecting Cq values of SARS-CoV-2 RT-PCR in the multivariate linear model, France, January -November $2020(\mathrm{n}=793,479)$

\begin{tabular}{|c|c|c|c|c|}
\hline Factor & Value & Coefficient & $2.5 \% \mathrm{Cl}$ & $97.5 \% \mathrm{Cl}$ \\
\hline \multicolumn{2}{|l|}{ Intercept } & 19.1 & 12.9 & $25 \cdot 4$ \\
\hline \multirow{2}{*}{ Assay } & PerkinElmer & \multicolumn{3}{|c|}{ Reference } \\
\hline & Genefinder & 12.1 & 10.3 & 13.9 \\
\hline \multirow{3}{*}{ Laboratory } & Lab_1 & \multicolumn{3}{|c|}{ Reference } \\
\hline & Lab_122 & $5 \cdot 42$ & 3.79 & 7.05 \\
\hline & Lab_96 & -4.8 & -6.71 & -2.90 \\
\hline \multirow{3}{*}{ Result } & Positive & \multicolumn{3}{|c|}{ Reference } \\
\hline & Weakly positive & 11.3 & 11.1 & 11.5 \\
\hline & Negative & 16.9 & 16.6 & 17.2 \\
\hline \multirow{3}{*}{ Days post symptom onset } & Less than 4 & \multicolumn{3}{|c|}{ Reference } \\
\hline & 4 to 7 & 2.76 & 2.66 & 2.86 \\
\hline & 8 to 14 & 4.90 & $4 \cdot 73$ & 5.08 \\
\hline \multirow{2}{*}{ Sample } & Nasopharyngeal & \multicolumn{3}{|c|}{ Reference } \\
\hline & Other & -1.81 & -2.49 & -1.14 \\
\hline Age & Per 20 years older & -0.541 & -0.585 & -0.497 \\
\hline \multirow{3}{*}{ Target gene } & $\mathrm{N}$ & \multicolumn{3}{|c|}{ Reference } \\
\hline & ORF1 & 1.03 & 0.949 & 1.12 \\
\hline & $\mathrm{S}$ & 1.19 & 0.948 & 1.43 \\
\hline Date & Per 71 days later & -0.797 & -0.903 & -0.691 \\
\hline
\end{tabular}

$\mathrm{Cl}$ : confidence interval; Cq: quantification cycle; ORF: open reading frame; SARS-CoV-2: severe acute respiratory syndrome coronavirus 2. We only list factors with significant effects with a 10-3 p value criterion. Coefficients reflect differences in Cq. For qualitative factors, the reference value is shown. See the Supplement for details about the model and the scaling of the quantitative variables.

generate testable hypotheses and help improve shortterm predictions for epidemic surveillance.

\section{Introduction}

Molecular testing is a key component of screening policies to control the spread of infectious diseases, and the coronavirus disease (COVID-19) pandemic has led to an unprecedented testing rate using reverse transcription PCR (RT-PCR) assays [1]. In clinical and public health practice, RT-PCR results are qualitative for viral respiratory disease diagnostics, with reports such as 'positive', 'negative', 'uninterpretable' and, sometimes, 'weakly positive'. These are based on the cycles threshold, also referred to as crossing point or crossing threshold (here denoted quantification cycles (Cq)), which corresponds to the number of PCR amplification cycles required for the fluorescent signal to rise above a positive threshold. In theory, the more abundant the genetic target in the sample, the fewer the amplification cycles required to detect it. This is why numerous studies on severe acute respiratory syndrome coronavirus 2 (SARS-CoV-2) rely on Cq values to assess transmissibility [2] or study infection kinetics [3]. However, many practical and biological limitations make Cq values a poor reflector of virus load [4].

Few studies analyse $\mathrm{Cq}$ values at a population level. One explanation is that these are known to suffer from several, potentially strong, biases. Firstly, sample type and sampling quality directly affect the amount of genetic material available. Secondly, the choice of RT-PCR assay matters. Even the quality of the reagents may have a considerable effect on the number of amplification cycles required to achieve the same level of fluorescence for the same amount of target genetic material. Combining data from different laboratories helps control for these sources of variation in statistical analyses. Furthermore, the larger the dataset, the more we can detect small statistical trends even after having controlled for non-informative variables.

Here, we present a cross-sectional analysis of SARSCoV-2 RT-PCR tests performed on samples from 2,220,212 individuals in France during the COVID-19 pandemic between 21 January and 30 November 2020 (Supplementary Figure S2 shows the daily number of tests and the number of tests per French department). Our aim was to determine firstly, if this analysis at the population level can identify cofactors of interests (e.g. age, sex, type of sample) and, therefore, new virological or immunological hypotheses and, secondly, if it can improve our ability to anticipate epidemic trends.

\section{Methods}

We studied SARS-CoV-2 RT-PCR tests performed in 2020 in France on samples from individuals aged between 1 and 89 years. The national French database for SARSCoV-2 RT-PCR tests (SI-DEP) collects qualitative results 
Correlations between key factors and observed Cq variations, SARS-CoV-2 RT-PCR tests, France, January-November 2020 $(\mathrm{n}=793,479)$

\section{A. Qualitative result of the test}

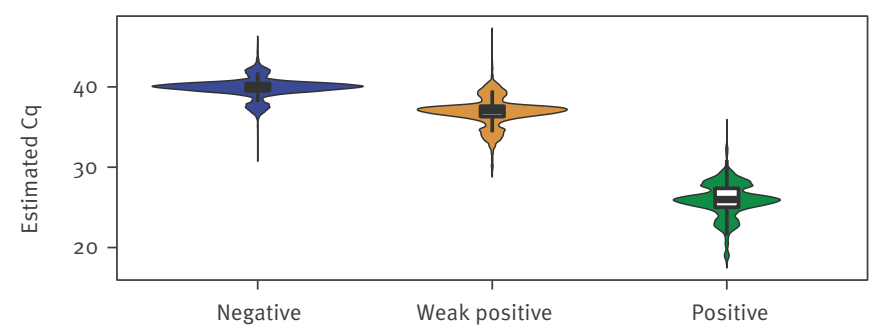

C. Participant age and sex

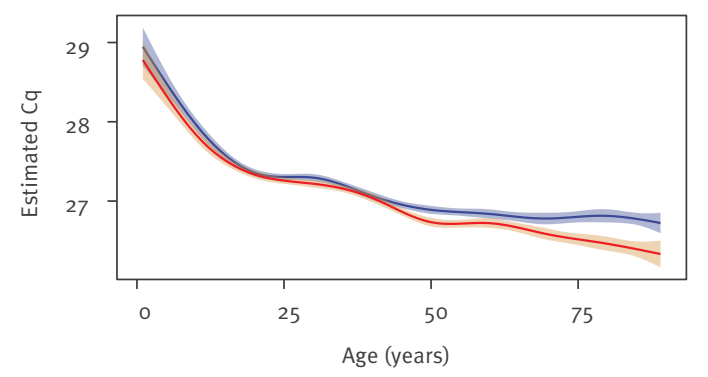

E. Sampling date

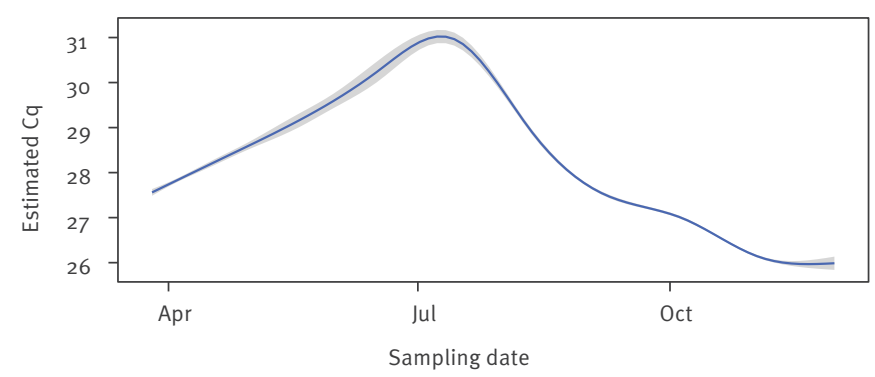

B. Number of days between symptoms onset and testing

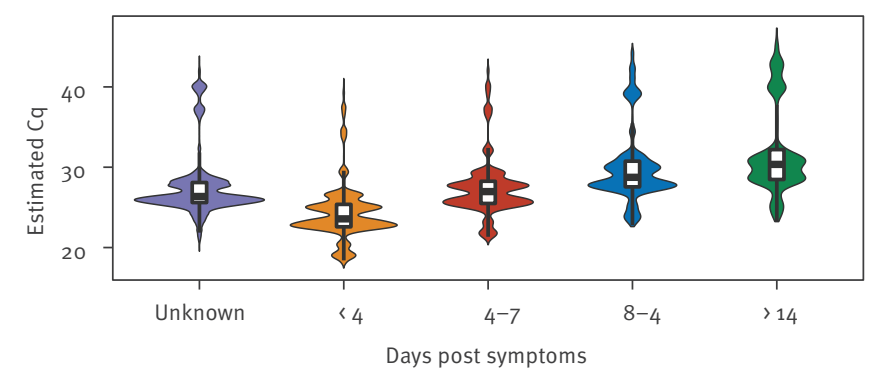

D. Genomic area targeted by the test

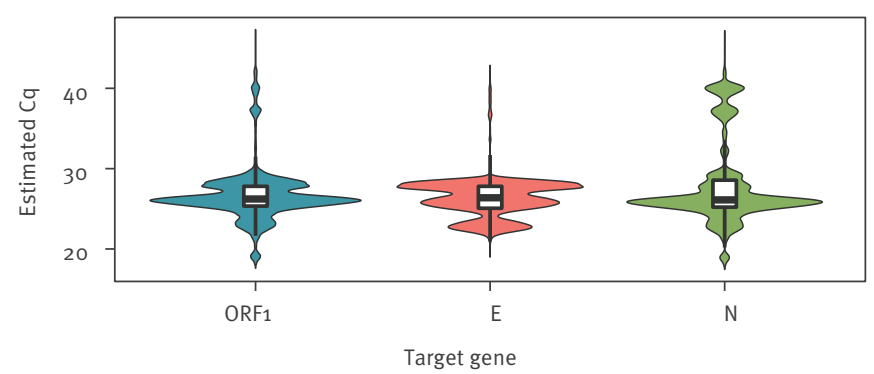

F. Temporal reproduction number at the time of the test

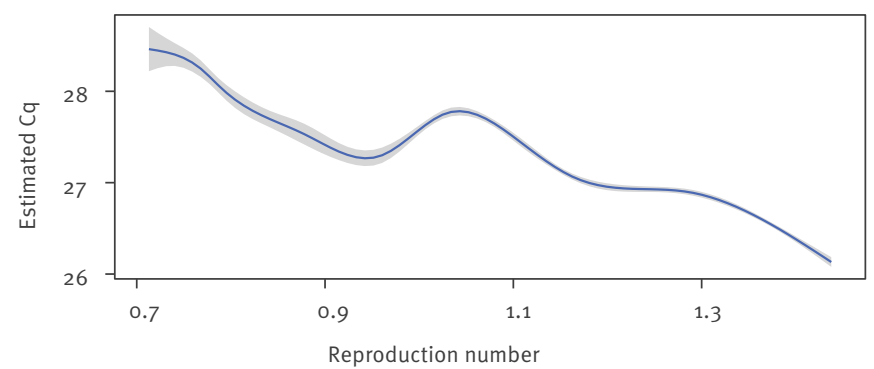

Cq: quantification cycle; F: female; M: male; ORF: open reading frame; SARS-CoV-2: severe acute respiratory syndrome coronavirus 2.

For panels A, B and D, the violin plots indicate the distributions and the box plots show the $0.025,0.25,0.5,0.75$ and 0.975 quantiles. Panels C, E and F are obtained with a 'loess' smoothing model and the grey area shows the $95 \%$ confidence interval from the underlying model. The Cq values shown are not the raw values but those estimated using a multiparametric linear model to correct for biases (see Supplementary Methods for details regarding the computation of the estimated values).

but $\mathrm{Cq}$ values are not reported. In order to focus on $\mathrm{Cq}$ values, this study relied on the French Society of Microbiology (SFM) network of hospital-based and private laboratories. Nationwide, databases from 21 laboratories, listed in the Supplement, were included on a voluntary basis. The geographical coverage of the tests is shown in Supplementary Figure S2B. The context in which these tests were performed varied over time. Until at least April 2020, the testing capacity was limited in the country and the majority of tests were performed on symptomatic individuals, especially in hospital settings. After May 2020, testing was more accessible and data then included screening tests performed in the general population. This change in testing context coincided with a shift in terms of screening facilities, with the majority of the tests being performed in hospital virology departments until April 2020 and in private laboratories after that. However, we do not expect that this shift led to a change in testing practice across French regions. We did not include tests for which key variables such as patient age, patient sex, laboratory geographical department, qualitative result or RT-PCR assay used were unknown. Note that one test could provide more than one $\mathrm{Cq}$ value if it contained probes targeting multiple viral genes. According to the national guidelines [5], it is recommended to focus on the most sensitive target to categorise levels of viral excretion. After removing the $388 \mathrm{Cq}$ values that we deemed marginal and potentially unrealistic because they were smaller than 10 or larger than 45 , the $95 \%$ confidence interval $(\mathrm{Cl})$ of the remaining values was 16.89-35.56 (details on the characteristics of the 


\section{FIGURE 2}

Predicting temporal reproduction number from time series related to SARS-CoV-2 RT-PCR tests, France, JanuaryNovember $2020(\mathrm{n}=330,611)$

\section{A. Positive test ratio}

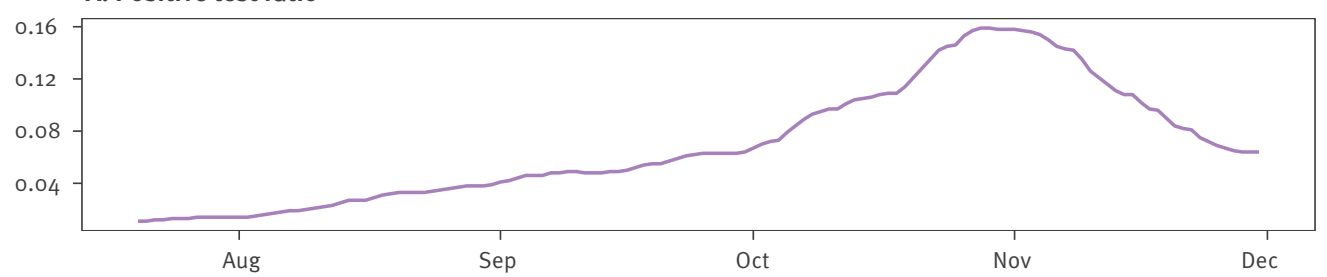

B. Cq median

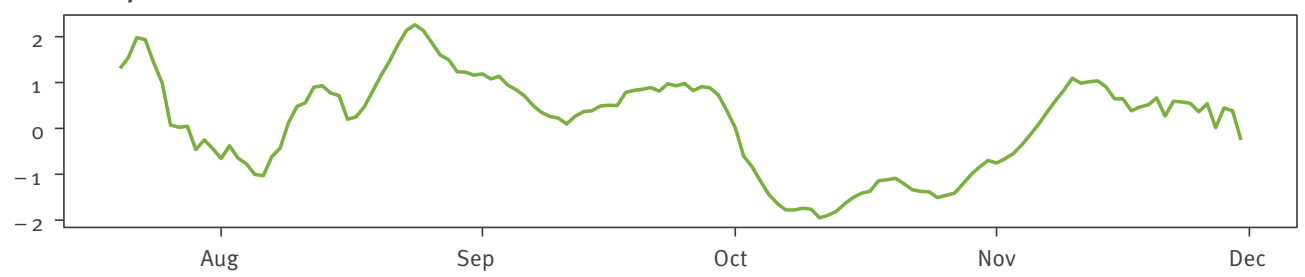

C. Cq skewness

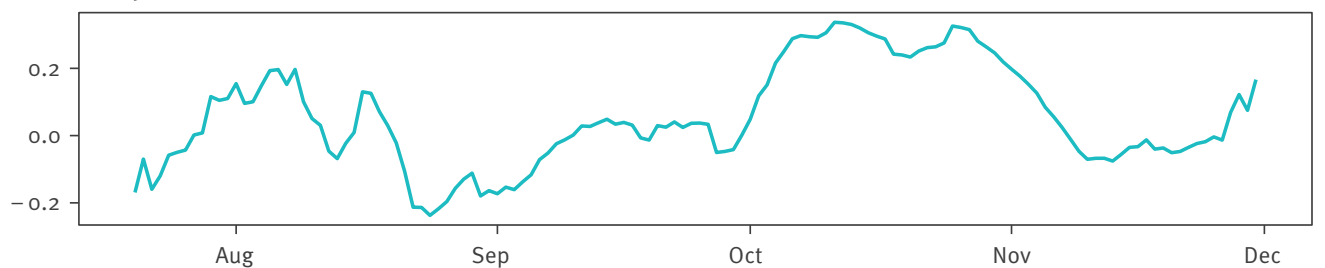

D. Temporal reproduction number

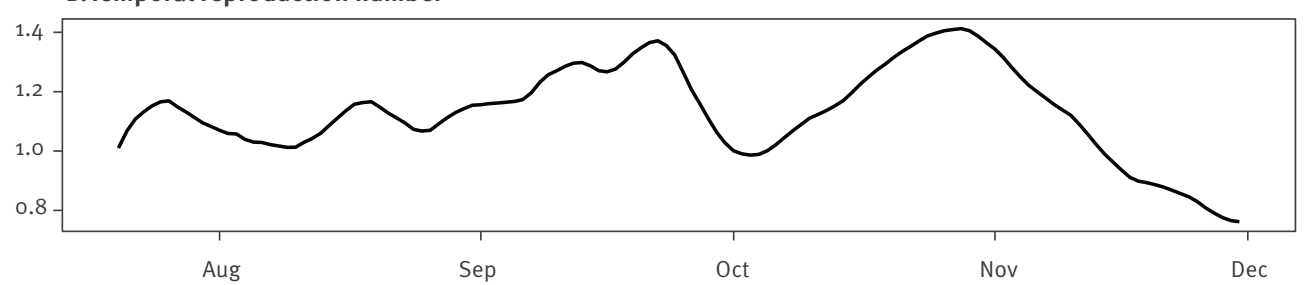

E. Mean absolute percentage error

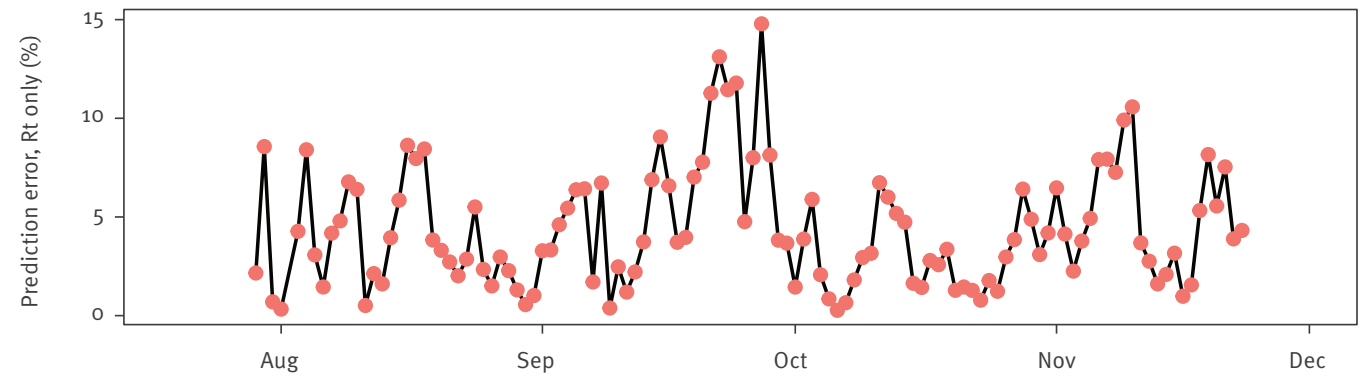

F. Difference in mean absolute percentage error

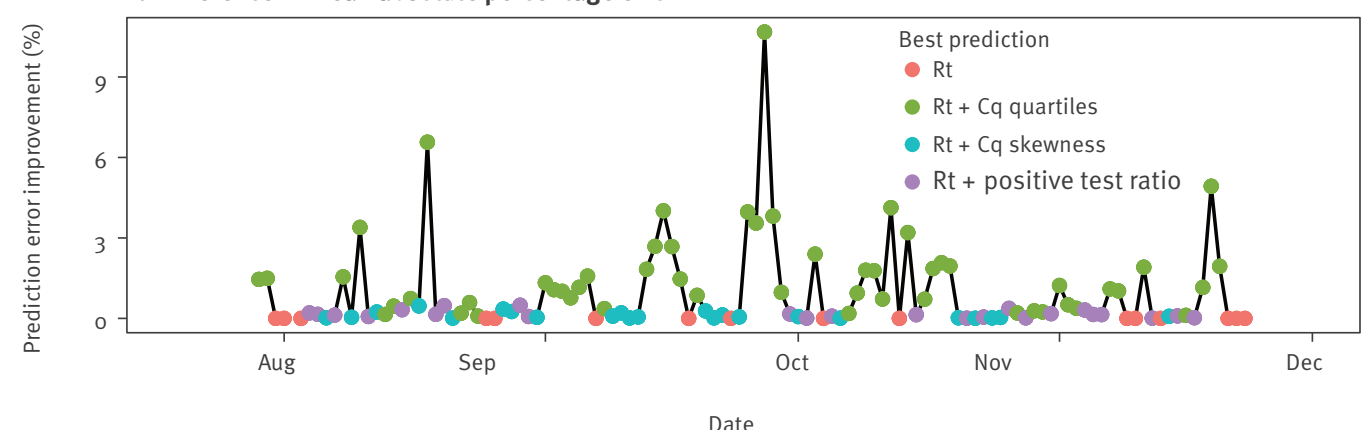

Cq: quantification cycle; Rt: temporal reproduction number; SARS-CoV-2: severe acute respiratory syndrome coronavirus 2.

Panels A-D show the the 7-day rolling averages of the time series of the ratio of positive tests (in purple), the median (in green) and skewness (in cyan) of the daily Cq residual distribution and Rt (in black). Panels E-F show the error made by a prediction using only Rt data (red dots) and the potential improvement made by including exogeneous data. 
whole dataset and its variables of interest are included in Supplementary Table S1). The median and upper bound of the $95 \% \mathrm{Cl}$ were unaffected by the removal of these values and the lower bound increased marginally from 16.87 to 16.89 . Overall, we were left with 793,479 tests from the same number of individuals, i.e. $35.7 \%$ of the whole database. The whole database contains tests with both negative and positive clinical results. However, we only kept tests with a Cq value and the former were less represented in the final database since samples that test negative usually do not have any reported $\mathrm{Cq}$ value (laboratories rarely record $\mathrm{Cq}$ values greater than 40 ).

We used a linear regression model to explore how $\mathrm{Cq}$ values can be explained by the following variables: patient age and sex, the number of days since the onset of symptoms (if known), the clinical sampling site (if known), the sampling facility (if known), the RT-PCR assay used, the target gene, the test's qualitative result, the sampling date, the temporal reproduction number $(R t)$ of the epidemic on the sampling date, and a control variable. The latter corresponds to the last digit of the patient anonymity number and is expected to be independent of the Cq value. Therefore, the lowest $p$ value associated with the control variable, which we expect to be pure noise, can be used to set the significance threshold for the other variables. We also included in the model an interaction term between sampling date and Rt. For this analysis, we excluded Cq values from internal controls. Univariate analyses are extremely sensitive to heterogeneity in the dataset. For instance, the age distribution from patients sampled in aged care homes is different from that in city screening facilities, and analysing the 'sampling facility' factor alone could yield misleading results. This is why the analysis used here was multivariate and considered all the factors listed above simultaneously. In particular, it allowed us to control for variations in the way the data were collected, e.g. the intensity or the context of the sampling.

To control for the consistency of the results for some of the factors, especially those related to the infection (e.g. the number of days since symptoms onset), we also performed the analyses only on the tests that were reported as 'positive' or 'weakly positive' (i.e. we ignored the tests labelled as negative by clinical virologists). These are shown in Supplementary Table S3.

The Rt, which can be interpreted as the number of secondary infections caused by a person infected at a given date $t$, was estimated using national hospital admission data and the EpiEstim method $[6,7]$. Furthermore, the time series analysis to explore the added value of $\mathrm{Cq}$ data was performed using autoregressive integrated moving average (ARIMA) models. Further details about the methods can be found in the Supplementary Methods.

\section{Ethical statement}

This study was approved by the Institutional Review Board of the Centre Hospitalier Universitaire de Montpellier. It is registered at ClinicalTrials.gov under the identifier NCTo4738331.

\section{Results}

Factors associated with Cq values

The adjusted R-square of the linear model was $38.8 \%$, meaning that the factors we chose explained one-third of the variance in $\mathrm{Cq}$ values. The model residuals were normally distributed (Supplementary Figure S3A). Care should be taken in the interpretation given that the data were unbalanced, which is why we performed an analysis of variance (ANOVA) with type II sums of squares. All factors except the Rt were significantly associated with $\mathrm{Cq}$ values using a classical $5 \% \mathrm{p}$ value criterion. Even for the control variable, the $p$ value was 0.013 , and patients with final digits 1 and 3 in their identification number had $\mathrm{Cq}$ values slightly lower (-0.19 and -0.17 cycles) than patients with a o as the final digit. Therefore, we set our significance thresholds to $5 \%$ of that of the control variables, i.e. $6.5 \times 10^{-4}$, to analyse the main effects (Table). Detailed outputs of the linear model are shown in Supplementary Table S2.

The intercept of the linear model indicates the average $\mathrm{Cq}$ value for a positive test performed with the reference assay, with all factors being set to their reference value. Its magnitude (19.1 cycles) is in line with clinical practice. The importance of the noise in the dataset is illustrated by the strong effect of the testing laboratory, as well as the RT-PCR assay used (Supplementary Figure $\mathrm{S}_{1}$ provides the distributions of $\mathrm{Cq}$ values as a function of the assay used and its target gene).

Despite this high level of noise, we detected a strong effect of the clinical qualitative result ('negative', 'positive' or 'weak positive') communicated by the testing laboratory (Figure $1 \mathrm{~A}$ ), with $\mathrm{Cq}$ differences that were even larger than those from the laboratory effect. We also found a significant difference of -1.81 cycles between the most common type of samples (nasopharyngeal) and other clinical sampling sites (mostly lower respiratory tract, but also faeces or saliva).

The effect associated with the number of days since symptom onset was particularly strong. The number of days between symptoms onset and testing dates was known for $8.5 \%$ of the participants; their Cq values increased gradually over the reported range of days with a maximum difference of 5.73 cycles (Figure 1B). The effect was similar when removing from the analysis the tests clinically considered to be 'negative' (see Supplementary Table S3 for these sensitivity analyses).

The effect of sex had the same order of magnitude as that of the control variable and could therefore be treated as non-significant. Conversely, the factor age 
had a strong effect, with a decrease of 0.541 cycles per 20.1 years of age increased (Figure $1 \mathrm{C}$ ).

The target gene of the RT-PCR assay used also yielded a significant effect. The $\mathrm{Cq}$ values obtained when using a probe targeting the ORF1 and $S$ regions of the virus genome were significantly higher than when using the $\mathrm{N}$ gene, which was the genomic region of reference in the model (Figure 1D).

Finally, we found that $\mathrm{Cq}$ values decreased with time ( -0.797 cycle per day), but this effect was nonlinear (Figure $1 \mathrm{E}$ ). This could be due to the strong variation in testing efforts in France (Supplementary Figure $\mathrm{S}_{2} \mathrm{~A}$ shows that the number of tests performed varied strongly during the year), but also to variations in the epidemic trend. Indeed, although the Rt (inferred from hospitalisation data) on the date of sampling was not significantly associated with the $\mathrm{Cq}$ value according to our threshold $\left(6.5 \times 10^{-4}\right.$, i.e. $5 \%$ of the lowest $p$ value of the control variables), the interaction between the sampling date and $R t$ was nearly significant with a $p$ value of $10^{-3}$ (Figure $1 \mathrm{~F}$ ), suggesting that a temporal analysis could yield additional insights.

\section{Anticipating epidemic spread using Cq values} The existence of a correlation between the $R t$ and the $\mathrm{Cq}$ values of the tests performed in a population is consistent with population dynamics theory, which predicts that in an expanding population of infected individuals, the 'age' of the infections, i.e. the number of days post infection, is skewed towards lower values [8]. Since $\mathrm{Cq}$ values have been reported to increase over the course of an infection [3], which we confirm with this analysis (Figure 1D), it has been suggested that these values could be used as an early signal to predict $R t$ [9].

To investigate this question, we focused on screening data collected in the general population only from individuals aged 5 to 80 years because younger or older individuals may be associated with specific epidemiological clusters, e.g. in daycare facilities or nursing homes. We estimated the median and skewness values of the daily distribution of the $\mathrm{Cq}$ values. To correct for potential confounding factors, these were adjusted using a linear model (see details in the Supplementary Methods). We analysed the temporal correlation between the time series with a 7-day rolling average of this median, skew and Rt (Figure 2). For the median Cq value, we found a significant correlation with $R t$ that was maximised for a delay of 6-7 days (Supplementary Figure $S_{4}$ provides additional information on the crosscorrelation functions between $R t$ and the median or the skewness of the $\mathrm{Cq}$ distribution). This is consistent with $R t$ being calculated using data from hospital admissions for COVID-19, which occur at a median of 10 to 14 days after infection $[10,11]$ and with RT-PCR screening data being obtained earlier in the infection. To further assess the usefulness of Cq data, we used ARIMA models to predict $R$ t dynamics over 7 days.
We compared models without any exogenous data to models that also included exogenous time series (either median or skewness of estimated $\mathrm{Cq}$ values distribution, or the fraction of positive tests [1]). As expected, the prediction error made using only endogenous data $(R t)$ was low in periods where $R t$ variations were limited. Furthermore, we found that adding exogenous data improved the prediction, especially when strong shifts in Rt were occurring (Figure 2). The $\mathrm{Cq}$ values tended to provide a better reduction in the error of the prediction than the ratio of positive tests.

\section{Discussion}

This analysis of a large database of RT-PCR tests performed in all of the French regions during the first two COVID-19 pandemic waves in 2020 confirms that population-level $\mathrm{Cq}$ values are noisy since even a linear model that featured 91 degrees of freedom did not explain the majority of the variance. However, owing to the law of large numbers, we detected several effects that are in line with biological observations and with virological properties. For instance, our finding that $\mathrm{Cq}$ values decreased as a function of the number of days after symptoms onset is consistent with longitudinal follow-up [3]. Another study also reported lower Cq values when the test was performed in symptomatic individuals [12]. The same study found that men had slightly lower $\mathrm{Cq}$ values than women, which was not significant in our analysis. Similarly, the difference we detected depending on the virus gene targeted by the RT-PCR assay used can be interpreted in the light of known differences in mRNA copy numbers between genes depending on their distance from the 3' end [13]. We also found slightly higher $\mathrm{Cq}$ values in samples collected from nasopharyngeal swabs compared with other samples (mainly lower respiratory tracts), but this is probably because the latter tests were performed in patients with more severe symptoms. Regarding the link we found between age and $\mathrm{Cq}$ values, although there are some mechanistic hypotheses as to why virus load would increase with age, such as variations in ACE2 receptor expression and immunosenescence [14], the evidence was mixed, with some studies reporting a decreasing trend $[15]$ and others not $[12,16]$. Here, using a multivariate approach on a large dataset allowed us to unravel a strong and significant decrease of Cq values with age.

Finally, we found $\mathrm{Cq}$ differences associated with the gene targeted by the RT-PCR assay that are consistent with the life cycle of the virus. As stressed by Michalakis et al. [4], since coronaviruses are (+)ssRNA viruses, they use the same RNA matrix for replication and transcription, both being amplified by diagnostic assays. The problem is that the RNA matrix for transmission is not the same for each gene as Coronaviridae transcripts can produce subgenomic mRNAs that lack part of the genome [17]. As a consequence, and as shown in cell cultures [13], genes at the 5' end of the genome are under-represented. This is consistent with our result where assays targeting the gene at the 3' end (the $\mathrm{N}$ 
gene) tend to have lower $\mathrm{Cq}$ values than assays targeting genes at the $5^{\prime}$ end (the ORF1 and $\mathrm{S}$ genes). Note that an alternative explanation could be that some probes target more conserved areas of the SARS-CoV-2 than others [18].

A limitation of our study is that although our dataset stands out by its size and its level of details, it is restricted to a single country where testing effort varied, both on a temporal and on a spatial scale (Supplementary Figure S2). Although the testing behaviour can be assumed to be homogeneous, epidemic spread was different between regions, which could blur the relationship between $\mathrm{Cq}$ values and $R t$ at the national level. Performing similar analyses in other European countries and regions can also be particularly informative. In our study, we chose to analyse all the tests performed that had a Cq value. This is debatable since high $\mathrm{Cq}$ values can be due to noise and this is the point of implementing cut-offs. However, $\mathrm{Cq}$ values are known to increase during the course of an infection [3] and these high values could correspond to patients detected in a late stage, which is expected to be more frequent in a declining epidemic [9]. To control for this potential bias, we also performed the analysis on a dataset without the tests with a 'negative' result. Finally, this analysis was conducted at the end of the year 2020 but since then, as in most countries, the emergence of variants has altered epidemiological dynamics in France $[19,20]$ and early reports suggest that the $\mathrm{Cq}$ value measured could depend on the variant causing the infection [21-25]. Vaccination has also changed the picture as indicated by Cq estimations in vaccine breakthrough infections $[24,25]$ and should be included as a host factor in future analyses.

As pointed out elsewhere, care should be taken when interpreting Cq values because of technical issues (different assays may yield higher or lower values) and biological issues (coronaviruses produce subgenomic RNAs of different lengths) [4]. However, in this analysis, we do not attempt to link $\mathrm{Cq}$ values to viral loads but rather analyse raw values at a population level. A promising output of this analysis is the possibility to use $\mathrm{Cq}$ values as an early signal to detect changes in epidemic behaviour, e.g. Rt values. Indeed, in 2020, our most robust descriptors of the epidemic on a short time scale originated from hospital admission data, but these still have a considerable delay relative to the status of the epidemic since patients are hospitalised 2 weeks after infection $[10,11]$. The ratio of positive tests performed in the population of interest can, in theory, provide earlier insights but it suffers from strong sampling biases. We show that accounting for population-based variations in $\mathrm{Cq}$ values can improve $R t$ predictions on a 7-day period. This is consistent with a recent study which found a correlation between $\mathrm{Rt}$ and $\mathrm{Cq}$ distribution skewness using data from nasopharyngeal specimens collected from staff and residents in four long-term care facilities in Massachusetts, United States [9]. Note that, contrarily to us, this earlier study did not factor in individual data such as patient age or symptomatic status, and it did not perform a cross-validation analysis that would control for temporal autocorrelation issues.

Our results show that analysing a large dataset of $\mathrm{Cq}$ values from screening tests can filter out the important amount of noise in these values. Inclusion of $\mathrm{Cq}$ values in routine surveillance calls for an adaptation to the current state of the epidemic, especially the evolution of variants and the increase in vaccination coverage, but also the integration with other types of data such as mobility data [26-28].

\section{Conclusion}

In many European Union countries, the qualitative outcome of SARS-CoV-2 screening tests are already aggregated in national databases to monitor epidemic spread. Adding $\mathrm{Cq}$ values as well as basic metadata (such as the RT-PCR assay used or, to a lesser extent, the age and sex) could be done while there should be minimal economical and ethical challenges. Our results call for a better integration of $\mathrm{Cq}$ values in national and European surveillance programmes to monitor epidemics caused by SARS-CoV-2 or other human viruses, especially since these data raise fewer ethical concerns than other sources such as mobility data.

\section{Acknowledgements}

We thank the ETE modelling team for discussion and the COVID-19 study group from the Société Française de Microbiologie (SFM) for their support.

\section{Conflict of interest}

None declared.

\section{Authors' contributions}

SA, VF, and SB conceived the study, SH-B, J-MG, LN, SP, TV, AdR, CT, MSo, RS, CB-B, MSa, A-SLH, SB, CL, JD, SH, CH, $\mathrm{DV}, \mathrm{HMD}, \mathrm{SF}, \mathrm{BV}, \mathrm{CH}, \mathrm{BL}, \mathrm{VF}$, and $\mathrm{SB}$ contributed anonymous data, SA compiled the anonymous data sets from the collaborating partners, SA, MTS, and CS analysed the data, SA wrote the first draft of the manuscript, all authors commented and approved the manuscript.

\section{References}

1. Hasell J, Mathieu E, Beltekian D, Macdonald B, Giattino C, Ortiz-Ospina E, et al. A cross-country database of COVID-19 testing. Sci Data. 2020;7(1):345. https://doi.org/10.1038/ S41597-020-00688-8 PMID: 33033256

2. He X, Lau EHY, Wu P, Deng X, Wang J, Hao X, et al. Temporal dynamics in viral shedding and transmissibility of COVID-19. Nat Med. 2020;26(5):672-5. https://doi.org/10.1038/s41591020-0869-5 PMID: 32296168

3. Néant N, Lingas G, Le Hingrat Q, Ghosn J, Engelmann I, Lepiller $\mathrm{Q}$, et al. Modeling SARS-CoV-2 viral kinetics and association with mortality in hospitalized patients from the French COVID cohort. Proc Natl Acad Sci USA. 2021;118(8):e2017962118. https://doi.org/10.1073/pnas.2017962118 PMID: 33536313

4. Michalakis Y, Sofonea MT, Alizon S, Bravo IG. SARS-CoV-2 viral RNA levels are not 'viral load'. Trends Microbiol. 
2021;29(11):970-2. https://doi.org/10.1016/j.tim.2021.08.008 PMID: 34535373

5. French Microbiology Society (SFM). Avis du 25 septembre 2020 de la Société Française de Microbiologie (SFM) relatif à l'interprétation de la valeur de Ct (estimation de la charge virale) obtenue en cas de RT-PCR SARS-CoV-2 positive sur les prélèvements cliniques réalisés à des fins diagnostiques ou de dépistage. [Opinion on 25 September 2020 of the French Society of Microbiology (SFM) relating to the interpretation of the Ct value (estimate of the viral load) obtained in the event of a positive SARS-CoV-2 RT-PCR on clinical samples taken for diagnostic or screening purposes]. Paris: SFM; 2021. French. Available from: https://www.sfm-microbiologie. org/wp-content/uploads/2021/01/Avis-SFM-valeur-Ctexcre\%CC\%81tion-virale-_-Version-def-14012021_V4.pdf

6. Cori A, Ferguson NM, Fraser C, Cauchemez S. A new framework and software to estimate time-varying reproduction numbers during epidemics. Am J Epidemiol. 2013;178(9):1505-12. https://doi.org/10.1093/aje/kwt133 PMID: 24043437

7. Thompson RN, Stockwin JE, van Gaalen RD, Polonsky JA, Kamvar ZN, Demarsh PA, et al. Improved inference of timevarying reproduction numbers during infectious disease outbreaks. Epidemics. 2019;29:100356. https://doi. org/10.1016/j.epidem.2019.100356 PMID: 31624039

8. Caswell H. Matrix population models: construction, analysis and interpretation. Sunderland: Sinauer Associates, Inc.; 1989.

9. Hay JA, Kennedy-Shaffer L, Kanjilal S, Lennon NJ, Gabriel $\mathrm{SB}$, Lipsitch M, et al. Estimating epidemiologic dynamics from cross-sectional viral load distributions. Science. 2021;373(6552):eabho635. https://doi.org/10.1126/science. abho635 PMID: 34083451

10. Sofonea MT, Reyné B, Elie B, Djidjou-Demasse R, Selinger C, Michalakis Y, et al. Memory is key in capturing COVID-19 epidemiological dynamics. Epidemics. 2021;35:100459. https://doi.org/10.1016/j.epidem.2021.100459 PMID: 34015676

11. Salje H, Tran Kiem C, Lefrancq N, Courtejoie N, Bosetti P, Paireau J, et al. Estimating the burden of SARS-CoV-2 in France. Science. 2020;369(6500):208-11. https://doi.org/10.1126/ science.abc3517 PMID: 32404476

12. Walker AS, Pritchard E, House T, Robotham IV, Birrell PI, Bell I, et al. Ct threshold values, a proxy for viral load in community SARS-CoV-2 cases, demonstrate wide variation across populations and over time. eLife. 2021;10:e64683. https://doi. org/10.7554/eLife.64683 PMID: 34250907

13. Finkel Y, Mizrahi O, Nachshon A, Weingarten-Gabbay S, Morgenstern D, Yahalom-Ronen Y, et al. The coding capacity of SARS-CoV-2. Nature. 2021;589(7840):125-30. https://doi. org/10.1038/S41586-020-2739-1 PMID: 32906143

14. Yang Y, Zhao Y, Zhang F, Zhang L, Li L. COVID-19 in elderly adults: clinical features, molecular mechanisms, and proposed strategies. Aging Dis. 2020;11(6):1481-95. https://doi. org/10.14336/AD.2020.0903 PMID: 33269102

15. Euser S, Aronson S. Manders, I Lelyveld Sv, Herpers B, Sinnige J, et al. SARS-CoV-2 viral load distribution reveals that viral loads increase with age: a retrospective cross-sectional cohort study. medRxiv. 2021.01.15.21249691. preprint. https://doi.or g/10.1101/2021.01.15.21249691 https://doi.org/10.1101/2021. 01.15 .21249691

16. Jones TC, Mühlemann B, Veith T, Biele G, Zuchowski M, Hofmann J, et al. An analysis of SARS-CoV-2 viral load by patient age. medRxiv. 2020.06.08.20125484. preprint. https://doi.org/10.1101/2020.06.08.20125484 https://doi.org/ 10.1101/2020.06.08.20125484

17. Ogando NS, Dalebout TJ, Zevenhoven-Dobbe JC, Limpens RWAL, van der Meer Y, Caly L, et al. SARS-coronavirus-2 replication in Vero E6 cells: replication kinetics, rapid adaptation and cytopathology. J Gen Virol. 2020;101(9):925-40. https://doi.org/10.1099/jgv.0.001453 PMID: 32568027

18. Dearlove B, Lewitus E, Bai H, Li Y, Reeves DB, Joyce MG, et al. A SARS-CoV-2 vaccine candidate would likely match all currently circulating variants. Proc Natl Acad Sci USA. 2020;117(38):23652-62. https://doi.org/10.1073/ pnas.2008281117 PMID: 32868447

19. Haim-Boukobza S, Roquebert B, Trombert-Paolantoni S, Lecorche E, Verdurme L, Foulongne V, et al. Detecting Rapid Spread of SARS-CoV-2 Variants, France, January 26-February 16, 2021. Emerg Infect Dis. 2021;27(5):1496-9. https://doi. org/10.3201/eid2705.210397 PMID: 33769253

20. Alizon S, Haim-Boukobza S, Foulongne V, Verdurme L, Trombert-Paolantoni S, Lecorche E, et al. Rapid spread of the SARS-CoV-2 Delta variant in some French regions, June 2021. Euro Surveill. 2021;26(28):2100573. https:// doi.org/10.2807/1560-7917.ES.2021.26.28.2100573 PMID: 34269174
21. Davies NG, Jarvis Cl, Edmunds WJ, Jewell NP, Diaz-Ordaz $\mathrm{K}$, Keogh RH, et al. Increased mortality in communitytested cases of SARS-CoV-2 lineage B.1.1.7. Nature. 2021;593(7858):270-4. https://doi.org/10.1038/s41586-02103426-1 PMID: 33723411

22. Faria NR, Mellan TA, Whittaker C, Claro IM, Candido DDS, Mishra S, et al. Genomics and epidemiology of the P.1 SARSCoV-2 lineage in Manaus, Brazil. Science. 2021;372(6544):81521. https://doi.org/10.1126/science.abh2644 PMID: 33853970

23. Roquebert B, Haim-Boukobza S, Trombert-Paolantoni S, Lecorche E, Verdurme L, Foulongne V, et al. SARS-CoV-2 variants of concern are associated with lower RT-PCR amplification cycles between January and March 2021 in France. medRxiv. 2021.03.19.21253971. preprint. https://doi.or g/10.1101/2021.03.19.21253971 https://doi.org/10.1101/2021. 03.19 .21253971

24. Brown CM, Vostok J, Johnson H, Burns M, Gharpure R, Sami S, et al. Outbreak of SARS-CoV-2 infections, including COVID-19 vaccine breakthrough infections, associated with large public gatherings - Barnstable County, Massachusetts, July 2021. MMWR Morb Mortal Wkly Rep. 2021;70(31):1059-62. https:// doi.org/10.15585/mmwr.mm7031e2 PMID: 34351882

25. Blanquart F, Abad C, Ambroise J, Bernard M, Cosentino G, Giannoli J-M, et al. Characterisation of vaccine breakthrough infections of SARS-CoV-2 Delta and Alpha variants and withinhost viral load dynamics in the community, France, June to July 2021. Euro Surveill. 2021;26(37):2100824. https:// doi.org/10.2807/1560-7917.ES.2021.26.37.2100824 PMID: 34533119

26. Kraemer MUG, Hill V, Ruis C, Dellicour S, Bajaj S, McCrone JT, et al. Spatiotemporal invasion dynamics of SARS-CoV-2 lineage B.1.1.7 emergence. Science. 2021;373(6557):889-95. https:// doi.org/10.1126/science.abj0113 PMID: 34301854

27. Pullano G, Di Domenico L, Sabbatini CE, Valdano E, Turbelin C, Debin M, et al. Underdetection of cases of COVID-19 in France threatens epidemic control. Nature. 2021;590(7844):134-9. https://doi.org/10.1038/s41586-020-03095-6 PMID: 33348340

28. Selinger C, Choisy M, Alizon S. Predicting COVID-19 incidence in French hospitals using human contact network analytics. Int J Infect Dis. 2021;111:100-7. https://doi.org/10.1016/j. ijid.2021.08.029 PMID: 34403783

\section{License, supplementary material and copyright}

This is an open-access article distributed under the terms of the Creative Commons Attribution (CC BY 4.0) Licence. You may share and adapt the material, but must give appropriate credit to the source, provide a link to the licence and indicate if changes were made.

Any supplementary material referenced in the article can be found in the online version.

This article is copyright of the authors or their affiliated institutions, 2022. 